\title{
Formación de nanocomplejos entre polifenoles de cáscaras de manzanas y beta-lactoglobulina para su potencial aplicación en alimentos funcionales
}

\author{
Nanocomplexes formation between polyphenols \\ of apple peels and beta-lactoglobulin for their potential \\ application in functional foods
}

\author{
Llopart, Emilce E. (1,2); Busti, Pablo A. (1); Verdini, Roxana A. (1,3); Delorenzi, Néstor J. (1). \\ (1) Facultad de Ciencias Bioquímicas y Farmacéuticas, Universidad Nacional de Rosario, Rosario, Santa Fe, Argentina. \\ (2) Consejo Nacional de Investigaciones Científicas y Técnicas (CONICET), Rosario, Santa Fe, Argentina. \\ (3) Instituto de Química Rosario (IQUIR, UNR-CONICET), Rosario, Santa Fe, Argentina.
}

Contacto: ndeloren@fbioyf.unr.edu.ar

RECIBIDO: 15/3/2018 APROBADO: 4/6/2018

\begin{abstract}
Resumen
Los polifenoles (PF) ejercen una importante influencia sobre la salud humana, principalmente por su capacidad antioxidante. Las manzanas poseen PF principalmente en su cáscara. La proteína mayoritaria del lactosuero, la beta-lactoglobulina $(\beta-\mathrm{lg})$, puede unir compuestos anfifílicos, como PF, protegiéndolos de agentes externos. Se estudió la posibilidad de vehiculizar PF de cáscara de manzanas rojas (Red Delicious), formando nanocomplejos (NC) con $\beta$-lg. El contenido de PF de cáscara de manzana roja ensayada fue de $266 \pm 2 \mathrm{mg}$ AG/100 g. Por ultrafiltración se determinó que el $57 \%$ formó $\mathrm{NC}$ con $\beta$-lg. Por medidas de extinción de la fluorescencia de los PF sobre $\beta$-lg se confirmó la formación de un complejo estático entre PF y $\beta$-lg. Las experiencias de extinción de la fluorescencia con acrilamida no mostraron diferencia significativa ante la presencia de PF, indicando que los PF se unen a un sitio distinto al del bolsillo hidrofóbico de la proteína. El tamaño de partícula y el potencial $\zeta$ de $\beta$-lg no se modificaron por agregado de PF. Se observó que la mayor parte de los PF forman NC con $\beta$-lg. Son necesarios más estudios para caracterizar el complejo con la finalidad de utilizarlo para enriquecer alimentos.

Palabras clave: Polifenoles, cáscaras de manzanas, beta-lactoglobulina, nanocomplejos.
\end{abstract}

\begin{abstract}
Polyphenols (PF) exert an important influence on human health, mainly due to their antioxidant capacity. Apples have PF mainly in their peel. The major whey protein, beta-lactoglobulin $(\beta-\lg )$, can bind amphiphilic compounds, such as PF, protecting them from the action of external agents. In this work, nanocomplexes (NC) of PF extracted from red apple peel (Red Delicious), and $\beta$-lg were studied. By ultrafiltration it was determined that $57 \%$ of the PF added bound to the protein. Fluorescence extinction of $\beta$-lg by PF addition performed at different temperatures confirmed the formation of a static complex. The fluorescence extinction of $\beta$-lg with acrylamide did not vary significantly in the presence of PF, indicating that PF bound to a site different from the hydrophobic pocket of the protein. Particle size and $\zeta$ potential measurements of $\beta$-lg were not significantly modified by the addition of PF. This fact pointed to a low number of PF bounded to the dimer of the protein. In conclusion, NC would allow PF vehiculization, their protection and use for enrichment functional foods.

Keywords: Polyphenols, apple peel, beta-lactoglobulin, nanocomplexes.
\end{abstract}

\section{Introducción}

Existe abundante evidencia científica que correlaciona la ingestión de polifenoles (PF) con una menor incidencia de enfermedades crónicas, tales como las cardiovasculares, diabetes y cáncer, debido a su actividad antioxidante (Landete, 2012; Del Río, et al., 2013; Lima, et al., 2014). Esto generó un creciente interés en aumentar el contenido de PF en la dieta, incorporándolos en alimentos funcionales (Benzie y Wachtel-Galor, 2012).

La manzana es una fruta ampliamente consumida. La Cámara Argentina de Fruticultores Integrados estima que la producción mundial de manzanas es de alrededor de 85 millones de toneladas (CAFI, 2017). Esta fruta posee, entre otros nutrientes, PF, y presenta el mayor contenido en la cáscara (Escarpa y González, 1998; Solovchenko y Schmitz-Eiberger, 2003; Wolfe, 
et al., 2003). Esta característica resultaría de interés para los productores, ya que podrían encontrar usos alternativos para los cultivos que no cumplen con los estándares de calidad y, por lo tanto, son considerados como descarte (Reyes y Cisneros-Zevallos, 2003; Jacobo-Velázquez y Cisneros-Zevallos, 2012).

La proteína mayoritaria del lactosuero, la betalactoglobulina $(\beta-\lg )$, es muy estudiada por su capacidad de ligar compuestos hidrofóbicos y anfifílicos como vitaminas liposolubles, ácidos grasos y PF (Livney, 2010; Livney, 2017). La interacción entre $\beta$-lg y PF del té ha sido muy estudiada, no así la interacción con PF extraídos de cáscara de manzanas, alimentos que presentan diferentes tipos y cantidades de PF (Rodríguez, et al., 2015). La interacción de la $\beta$-lg con estos compuestos está regida principalmente por interacciones en su bolsillo hidrofóbico, pero también se han descripto enlaces a través de otros sitios presentes en la cavidad cercana a la estructura de alfa-hélice y en la superficie externa de la molécula (Kontopidis, et al., 2004; Delorenzi, 2005).

La incorporación de sustancias bioactivas a alimentos es una manera de desarrollar productos alimenticios funcionales que pueden proveer beneficios fisiológicos y/o disminuir el riesgo de contraer enfermedades (Chen y Subirade, 2006). Con el fin de incorporar sustancias bioactivas a alimentos a través de técnicas nanotecnológicas, el proceso de encapsulación presenta gran potencial para el desarrollo de productos innovadores en la industria alimentaria (Sekhon, 2010). Al atrapar un bioactivo dentro de un núcleo encapsulante se lo protege de posibles condiciones adversas ( $\mathrm{pH}$, luz, temperatura, humedad u oxígeno), preservando su funcionalidad, aumentando su vida útil y posibilitando su transporte (Tavares, et al., 2014; Quintanilla-Carvajal, et al., 2010).

Por lo antedicho, se propuso evaluar la posibilidad de vehiculizar PF de cáscara de manzanas rojas (Red Delicious), formando nanocomplejos (NC) con $\beta$-lg.

\section{Materiales y Métodos}

\section{Reactivos}

Se utilizó $\beta$-lg DAVISCO Foods International, Inc. (Le Sueur, $\mathrm{MN})$. Se preparó una solución madre de $\beta$-lg $100 \mu \mathrm{M}$ en buffer fosfato $20 \mathrm{mM}$ (pH 6,8). La solución de acrilamida (Sigma, USA) $5 \mathrm{M}$ se preparó en buffer fosfato $20 \mathrm{mM}$ (pH 6,8). Todos los demás productos químicos fueron de calidad analítica.

\section{Manzanas}

Se adquirieron manzanas Red Delicious que se lavaron, escurrieron y secaron con papel. Luego se procedió a pelarlas y, a partir de la cáscara, extraer los PF.

\section{Extracción de PF}

Los PF de las manzanas fueron extraídos por un método adaptado del trabajo de Wolfe et al. (2003). Se mezclaron $5 \mathrm{~g}$ de cáscara de manzana trozada con $20 \mathrm{~mL}$ de una solución fría de metanol/agua 80:20 durante $5 \mathrm{~min}$. La preparación se homogeneizó durante $3 \mathrm{~min}$. Luego se filtró a través de papel Whatman $\mathrm{N}^{\circ} 1$ y los sólidos se mezclaron con $15 \mathrm{~mL}$ de metanol 80\%. Se homogeneizó nuevamente por 3 min antes de filtrar. El filtrado se recuperó y se evaporó hasta sequedad usando un evaporador rotatorio Buchi (Alemania) a $45^{\circ} \mathrm{C}$.
Los extractos se reconstituyeron en $3,5 \mathrm{~mL}$ con buffer fosfato $20 \mathrm{mM} \mathrm{pH} 6,8$ y se congelaron a $-40^{\circ} \mathrm{C}$ hasta su análisis. Todos los extractos se realizaron por triplicado.

\section{Determinación del contenido de PF}

Se determinó el contenido de PF por la técnica de Folin-Ciocalteau (Singleton, et al., 1999) usando una curva patrón de ácido gálico $(\mathrm{AG})$ en un rango de concentración de 0 a 500 $\mu \mathrm{g} / \mathrm{mL}$. Los resultados se expresaron como equivalentes de AG g/100g de muestra seca.

\section{Determinación de formación \\ de complejos de $\beta$-lg con \\ PF por ultrafiltración}

El porcentaje de PF unidos a $\beta$-lg fue determinado por ultrafiltración con un equipo Amicon Modelo 1275 PSI max provisto de una membrana de filtración molecular de celulosa MWCO 10,000. En el ultrafiltrado el ligando libre se midió por Folin-Ciocalteau y el porcentaje de ligando fijado se calculó según la siguiente fórmula: $\mathrm{PF}$ unidos $(\%)=(\mathrm{PF}$ total $-\mathrm{PF}$ en el filtrado $) / \mathrm{PF}$ total $\mathrm{x} 100$

\section{Extinción de la fluorescencia intrínseca de la $\beta$-lg por PF}

Esta extinción fue estudiada usando una solución de proteína $20 \mu \mathrm{M}$ en buffer fosfato $20 \mathrm{mM}, \mathrm{pH} 6,8$. Se colocaron alícuotas de $3 \mathrm{~mL}$ de esta solución en la cubeta de un espectrofluorómetro Jasco FP-770 y se tituló con una solución madre de PF de $210 \mu \mathrm{M}$. La intensidad de fluorescencia a $336 \mathrm{~nm}$ fue medida después de cada adición de solución de PF utilizando una excitación de $295 \mathrm{~nm}$. Todas las experiencias fueron llevadas a cabo a dos temperaturas: $25^{\circ} \mathrm{C}$ y $35^{\circ} \mathrm{C}$.

Las correspondientes constantes de Stern-Volmer (Ksv) fueron obtenidas a partir de las pendientes de las gráficas Fo/F vs. concentración de agente extintor, de acuerdo con la siguiente ecuación (Liang y Subirade, 2012):

Fo/F $=1+$ Ksv [extintor]

Fo y $\mathrm{F}$ son la fluorescencia en ausencia y presencia de extintor.

\section{Extinción de la fluorescencia intrínseca de la $\beta$-lg y por acrilamida}

La extinción fue estudiada usando una solución de proteína $20 \mu \mathrm{M}$, en buffer fosfato $20 \mathrm{mM}, \mathrm{pH} 6,8$, en ausencia y presencia de $100 \mu \mathrm{l}$ de PF $210 \mu \mathrm{M}$ de cáscara de manzana. Se colocaron alícuotas de $3 \mathrm{~mL}$ de esta solución en la cubeta de un espectrofluorómetro Jasco FP-770 y se tituló con una solución madre de acrilamida de $5 \mathrm{M}$. La intensidad de fluorescencia a $336 \mathrm{~nm}$ fue medida después de cada adición de alícuota de acrilamida con una excitación de $295 \mathrm{~nm}$. Todas las experiencias fueron llevadas a cabo a $25^{\circ} \mathrm{C}$. Las constantes de Stern-Volmer (Ksv) se obtuvieron de manera similar a lo descripto en la sección anterior.

\section{Mediciones de tamaño de partícula y potencial $Z$}

Las mediciones del tamaño de partícula fueron llevadas a cabo mediante el uso de un instrumental que mide la dispersión 
dinámica de la luz (DLS) (Horiba, Nano Particle Analyzer SZ-100, Kyoto, Japan). El diámetro hidrodinámico es el diámetro de una esfera que difunde a la misma velocidad promedio que la partícula bajo investigación. El diámetro hidrodinámico medio (d) es el pico obtenido de la curva que representa la intensidad versus el diámetro de partícula. La carga eléctrica (potencial $\zeta$ ) de la proteína en presencia de PF fue determinada usando medidas electroforéticas. Antes de la determinación, las muestras fueron filtradas a través de microfiltros de $1 \mu \mathrm{m}$ de tamaño de poro. Las medidas de DLS y potencial $\zeta$ fueron llevadas a cabo sobre soluciones de $\beta$-lg $100 \mu \mathrm{M}$, en ausencia y presencia de cantidades variables de $210 \mu \mathrm{M}$ PF de cáscaras de manzanas. Las medidas se efectuaron por triplicado. La temperatura se mantuvo en $25^{\circ} \mathrm{C}$.

\section{Análisis estadístico}

Todas las determinaciones se realizaron al menos por triplicado y se informaron los promedios y desvíos correspondientes. Para la realización del análisis de varianza se utilizó el software estadístico Statgraphics plus 3.0.

\section{Resultados}

El contenido de PF de la cáscara de manzana roja Red Delicious obtenido fue de $266 \pm 2 \mathrm{mg} \mathrm{AG/100} \mathrm{g}$. Por ultrafiltración se determinó luego que el $57 \%$ del total de los PF presentes se ligó a la $\beta$-lg.

El análisis de la extinción de la fluorescencia intrínseca de la $\beta$-lg por PF presentó una constante de Stern-Volmer de $0,11 \mathrm{M}^{-1}$ a $25^{\circ} \mathrm{C}$ y $0,08 \mathrm{M}^{-1}$ a $35^{\circ} \mathrm{C}$, y fue significativamente menor la última $(p<0,05)$ (Figura $1 \mathrm{~A}$ ).
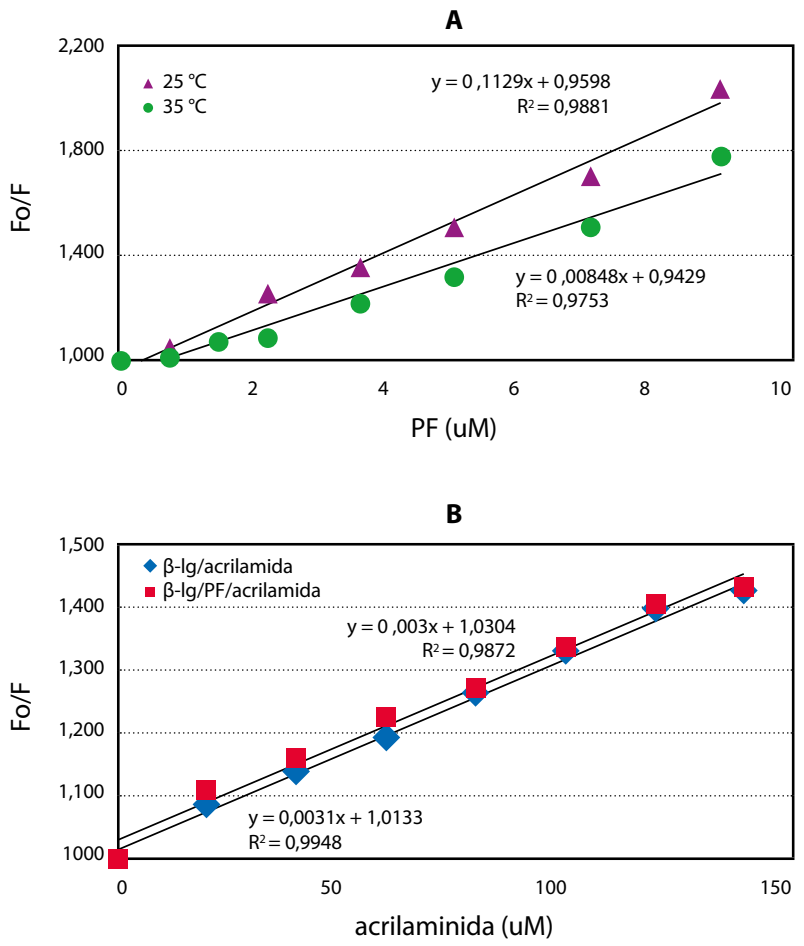

Figura 1. A) Extinción de la fluorescencia de $\beta$-lg por PF. B) Extinción de la fluorescencia de la $\beta$-lg con acrilamida en ausencia y presencia de PF.

\begin{tabular}{|c|c|}
\hline Sistema & Tamaño de partícula (nm) \\
\hline$\beta-\lg$ & $4,98 \pm 0,97$ \\
$\beta-\lg +0,4 \mu \mathrm{M} P F$ & $5,30 \pm 0,67$ \\
$\beta-\lg +0,7 \mu \mathrm{M}$ PF & $4,88 \pm 1,11$ \\
$\beta-\lg +1,1 \mu \mathrm{M}$ PF & $4,70 \pm 0,94$ \\
$\beta-\lg +1,5 \mu \mathrm{M}$ PF & $5,36 \pm 0,90$ \\
$\beta-\lg +1,9 \mu \mathrm{M} P F$ & $5,42 \pm 0,96$ \\
$\beta-\lg +2,2 \mu \mathrm{M}$ PF & $5,42 \pm 0,93$ \\
$\beta-\lg +3,7 \mu \mathrm{M}$ PF & $5,36 \pm 1,02$ \\
$\beta-\lg +4,5 \mu \mathrm{M} \mathrm{PF}$ & $4,62 \pm 0,74$ \\
$\beta-\lg +6,0 \mu \mathrm{M}$ PF & $4,24 \pm 0,96$ \\
$\beta-\lg +7,4 \mu \mathrm{M}$ PF & $4,62 \pm 0,61$ \\
\hline
\end{tabular}

Tabla 1. Tamaño de partícula de $\beta$-lg y complejos de PF con $\beta$-lg. Los resultados se expresaron como $\mathrm{x} \pm \mathrm{DE}$.

\begin{tabular}{|c|c|}
\hline Sistema & Potencial $\zeta(\mathrm{mV})$ \\
\hline$\beta$-LG & $-12,89 \pm 1,18$ \\
$\beta-\mathrm{LG}+0,6 \mu \mathrm{M}$ PF & $-11,87 \pm 1,09$ \\
$\beta-\mathrm{LG}+1,2 \mu \mathrm{M}$ PF & $-12,45 \pm 1,50$ \\
$\beta-\mathrm{LG}+1,7 \mu \mathrm{M}$ PF & $-11,98 \pm 0,78$ \\
$\beta-\mathrm{LG}+2,3 \mu \mathrm{M}$ PF & $-11,48 \pm 3,09$ \\
$\beta-\mathrm{LG}+2,9 \mu \mathrm{M}$ PF & $-12,60 \pm 1,79$ \\
$\beta-\mathrm{LG}+3,5 \mu \mathrm{M}$ PF & $-12,65 \pm 0,15$ \\
$\beta-\mathrm{LG}+4,6 \mu \mathrm{M}$ PF & $-12,20 \pm 0,77$ \\
\hline
\end{tabular}

Tabla 2. Potencial $\zeta$ de $\beta$-lg y complejos de PF con $\beta$-lg. Los resultados se expresaron como $\mathrm{x} \pm \mathrm{DE}$.

Al realizarse las experiencias de extinción de la fluorescencia con acrilamida de $\beta$-lg, en ausencia y presencia de PF, no se observó diferencia significativa $(p>0,05)$ (Figura 1B).

La $\beta$-lg mostró una distribución monomodal que se ensancha de 3,00 a $6,80 \mathrm{~nm}$, con un pico máximo a $25^{\circ} \mathrm{C}(4,98$ $\mathrm{nm})$. Cuando se agregaron concentraciones crecientes de PF, no se observó ningún cambio significativo en el tamaño de partícula $(p>0,05)$ (Tabla 1$)$.

La adición de PF de cáscara de manzana no modificó en forma significativa el potencial $\zeta$ de la $\beta-\lg (p>0,05)$ (Tabla 2).

\section{Discusión y Conclusión}

El valor hallado para el contenido de PF de la cáscara de manzana roja Red Delicious es cercano al publicado por Jakobek y Barron (2016), quienes trabajando con manzanas de piel roja obtuvieron contenidos de PF de entre 184 y 417 mg AG/100 g. Otros autores, trabajando con diferentes variedades de 
manzanas, hallaron contenidos de entre 165 y 400 mg AG/ 100 g (Valavanidis, et al., 2009), 206 y 472 mg AG/100 g (Wang, et al., 2015), 305 y $713 \mathrm{mg} \mathrm{AG/100} \mathrm{g} \mathrm{(Vieira,} \mathrm{et} \mathrm{al.,} \mathrm{2011),} \mathrm{y}$ 309 y 598 mg AG/100 g (Wolfe, et al., 2003).

Rodríguez et al. (2015) estudiaron por ultrafiltración el porcentaje de PF de té verde unidos a $\beta$-lg y obtuvieron valores de entre 62 y $85 \%$, moderadamente superiores a los hallados en este trabajo.

La extinción de la fluorescencia de los PF sobre $\beta-\lg$ mostró una reducción de la constante de Stern-Volmer al aumentar la temperatura, lo cual confirma la formación de un complejo estático entre $\beta$-lg y PF (Lakowicz, 2006). Por otra parte, el hecho de que no se observa diferencia en la extinción de la fluorescencia de la $\beta$-lg con acrilamida en ausencia y presencia de los PF indicaría que los PF se unirían a un sitio distinto al del bolsillo hidrofóbico sin producir cambios conformacionales evidentes (Moro, et al., 2001). Contrariamente, Kanakis et al. (2011), quienes estudiaron la interacción de $\beta$-lg con PF del té, observaron que estos se unen débilmente a la proteína en solución por interacciones hidrofóbicas e hidrofílicas, produciendo notables cambios conformacionales.

El tamaño de partícula determinado para la $\beta$-lg nativa estuvo de acuerdo con los datos previos informados por Von Staszewski et al. (2012) y Von Staszewski et al. (2014). En el rango de trabajo ensayado, el complejo entre la proteína y los PF involucró al dímero de $\beta$-lg, tal como lo evidencia la Tabla 1. Asimismo, la unión de PF cargados no fue suficiente para modificar la carga superficial de la proteína e indicó un número relativamente bajo de los PF unidos a la proteína (Tabla 2).

Utilizando PF de distinto origen (PF de té verde) y a concentraciones superiores a las de este trabajo, Rodríguez et al. (2015) observaron la formación de estructuras multiméricas. Bajo estas condiciones, otros autores suponen también un apilamiento de PF sobre zonas hidrofóbicas de la proteína debido a su estructura planar (Charlton, et al., 2002; Poncet-Legrand, et al., 2006; Von Staszewski, et al., 2014). La unión entre proteínas del lactosuero y $\mathrm{PF}$ se ha caracterizado como interacciones de enlace no covalente, que comprenden interacción hidrofóbica, van der Waals, puente de hidrógeno e interacción iónica (Nagy, et al., 2012).

La formación de NC entre los PF de cáscara de manzanas y dímeros de la $\beta$-lg como modelo de proteínas del lactosuero permitiría su vehiculización, protección y posterior utilización para el enriquecimiento de alimentos. Para tal fin se deberían realizar estudios de estabilidad de los NC ante la acción de agentes externos y de biodisponibilidad. Además, debería evaluarse la aceptabilidad sensorial de los alimentos enriquecidos.

\section{Referencias}

Benzie, I.F. y Wachtel-Galor, S., 2012. Increasing the antioxidant content of food: A personal view on whether this is possible or desirable. En: International Journal of Food Sciences and Nutrition, 63(1), pp.62-70.

CAFI- Cámara Argentina de Fruticultores Integrados, 2017. Mercado mundial de la manzana [En línea]. Río Negro: CAFI. [Consulta: 10-02-2018]. Disponible en: http:// www.cafi.org.ar/mercado-mundial-de-la-manzana/

Charlton, A.J., Baxter, N.J., Lokman Khan, M., Moir, A.J.G., Haslam, E., Davies, A.P., et al., 2002. Polyphenol/peptide binding and precipitation. En: Journal of Agricultural and Food Chemistry, 50, pp.1593-1601.

Chen, L. y Subirade, M., 2006. Alginate-whey protein granular microspheres as oral delivery vehicles for bioactive compounds. En: Biomaterials, 27(26), pp.4646-4654.

Del Río, D, Rodriguez-Mateos, A, Spencer, JPE, Tognolini, M, Borges, G. y Crozier, A, 2013. Dietary (poly) phenolics in human health: structures, bioavailability, and evidence of protective effects against chronic diseases. En: Antioxidants $y$ Redox Signaling, 18(14), pp.1818-1892.

Delorenzi, N. J., 2005. Beta-lactoglobulin conformational changes studied by fluorescence quenching. En: Riley, Arthur P., ed., 2005. Food research, safety and policies. Cap.7. New York: Nova Science Publishers. pp.159-174.

Escarpa, A. y González, M.C., 1998. High-performance liquid chromatography with diode-array detection for the determination of phenolic compounds in peel and pulp from different apple varieties. En: Journal of Chromatography, 823, pp.331-337.

Jacobo-Velázquez, D.A. y Cisneros-Zevallos, L., 2012. An alternative use of horticultural crops: stressed plants as biofactories of bioactive phenolic compounds. En: Agriculture, 2(3), pp.259-271.

Jakobek, L. y Barron, A.R., 2016. Ancient apple varieties from Croatia as a source of bioactive polyphenolic compounds. En: Journal of Food Composition and Analysis, 45, pp.9-15.

Kanakis, C.D., Hasni, I., Bourassa, P., Tarantilis, P.A., Polissiou, M.G. y Tajmir-Riahi, H.A., 2011. Milk $\beta$-lactoglobulin complexes with tea polyphenols. En: Food Chemistry, 127(3), pp.1046-1055.

Kontopidis, G., Holt, C. y Sawyer, L., 2004. Beta-lactoglobulin: binding properties, structure, and function. En: Journal of Dairy Science, 87, pp.785-796.

Lakowicz, J.R., 2006. Quenching of fluorescence. En: Lakowicz, Joseph R. Principles of fluorescence spectroscopy. 3rd ed. Cap.8. New York: Springer. pp.323-350.

Landete, J.M., 2012. Updated knowledge about polyphenols: functions, bioavailability, metabolism, and health. En: Critical Reviews in Food Science and Nutrition, 52(26), pp. 936-948.

Liang, L. y Subirade, M., 2012. Study of the acid and thermal stability of blactoglobulin-ligand complexes using fluorescence quenching. En: Food Chemistry, 132(4), pp.2023-2029.

Lima, G.P.P., Vianello, F., Corrêa, C.R., da Silva Campos, R.A. y Borguini, M.G., 2014. Polyphenols in Fruits and Vegetables and Its Effect on Human Health. En: Food and Nutrition Sciences, 5(11), pp.1065.

Livney, Y.D., 2010. Milk proteins as vehicles for bioactives. En: Current Opinion in Colloid \& Interface Science, 15, pp.7383.

Livney, Y.D., 2017. Nanoencapsulation technologies. En: Roos, Yrjö H. y Livney, Yoav D., eds. Engineering foods for bioactives stability and delivery. New York: Springer. pp.143-169.

Moro, A., Gatti, C.A. y Delorenzi, N.J., 2001. Hydrophobicity of whey protein concentrates measured by fluorescence quenching and its correlation with surface functional properties. En: Journal of Agricultural and Food Chemistry, 49, pp.4784-4789.

Nagy, K., Courtet-Compondu, M.C., Williamson, G., Rezzi, S., Kussmann, M. y Rytz, A., 2012. Non-covalent binding of proteins to polyphenols correlates with their amino acid sequence. En: Food Chemistry, 132, pp.1333-1339. 
Poncet-Legrand, C., Edelmann, A., Putaux, J.L., Cartalade, D., Sarni-Manchado, P. y Vernhet, A., 2006. Poly (L-proline) interactions with flavan-3-ols units: Influence of the molecular structure and the polyphenol/protein ratio. En: Food Hydrocolloids, 20(5), pp.687-697.

Quintanilla-Carvajal, M.X., Camacho-Díaz, B.H., MerazTorres, L.S., Chanona-Pérez, J.J., Alamilla-Beltrán, L., Jiménez Aparicio, A. y Gutiérrez-López, G.F., 2010. Nanoencapsulation: a new trend in food engineering processing. En: Food Engineering Reviews, 2(1), pp.39-50.

Reyes, L.F. y Cisneros-Zevallos, L., 2003. Wounding stress increases the phenolic content and antioxidant capacity of purple-flesh potatoes (Solanum tuberosum L.). En: Journal of Agricultural and Food Chemistry, 51(18), pp.5296-5300.

Rodríguez, S.D., Von Staszewski, M. y Pilosof, A.M., 2015. Green tea polyphenols-whey proteins nanoparticles: Bulk, interfacial and foaming behavior. En: Food Hydrocolloids, 50, pp.108-115.

Sekhon, B.S., 2010. Food nanotechnology e an overview. En Nanotechnology, Science and Applications, (3), pp.1-15.

Singleton, V.L., Orthofer, R. y Lamuela-Raventos, R.M., 1999. Analysis of total phenols and other oxidation substrates and antioxidants by means of the Folin-Ciocalteu reagent En: Methods in Enzymology, 299, pp.152-178.

Solovchenko, A. y Schmitz-Eiberger, M., 2003. Significance of skin flavonoids for UV-B-protection in apple fruits. En: Journal of Experimental Botany, 54, pp.1977-1984.

Tavares, G.M., Croguennec, T., Carvalho, A.F. y Bouhallab, S., 2014. Milk proteins as encapsulation devices and delivery vehicles: Applications and trends. En: Trends in Food Science \& Technology, 37, pp.5-20.

Valavanidis, A., Vlachogianni, T., Psomas, A., Zovoili, A. y Siatis, V., 2009. Polyphenolic profile and antioxidant activity of five apple cultivars grown under organic and conventional agricultural practices. En: International Journal of Food Science \& Technology, 44(6), pp.1167-1175.

Vieira, F.G.K., Borges, G.D.S.C., Copetti, C., Di Pietro, P.F., da Costa, Nunes, E. y Fett, R., 2011. Phenolic compounds and antioxidant activity of the apple flesh and peel of eleven cultivars grown in Brazil. En: Scientia Horticulturae, 128(3), pp.261-266.

Von Staszewski, M., Jara, F.L., Ruiz, A.L., Jagus, R.J., Carvalho, J.E. y Pilosof, A.M., 2012. Nanocomplex formation between $\beta$-lactoglobulin or caseinomacropeptide and green tea polyphenols: Impact on protein gelation and polyphenols antiproliferative activity. En: Journal of functional foods, 4(4), pp.800-809.

Von Staszewski, M., Ruiz-Henestrosa, V.M.P. y Pilosof, A.M., 2014. Green tea polyphenols- $\beta$-lactoglobulin nanocomplexes: Interfacial behavior, emulsification and oxidation stability of fish oil. En: Food Hydrocolloids, 35, pp.505-511.

Wang, X., Li, C., Liang, D., Zou, Y., Li, P. y Ma, F., 2015. Phenolic compounds and antioxidant activity in redfleshed apples. En: Journal of Functional Foods, 18 pp.1086-1094.

Wolfe, K., Wu, X. y Liu, R.H., 2003. Antioxidant activity of apple peels. En: Journal of Agricultural and Food Chemistry, 51(3), pp.609-614. 